\title{
Addendum to West et al Addendum: Paroxetine-Induced Increase in Activity of Locus Coeruleus Neurons in Adolescent Rats: Implication of a Countertherapeutic Effect of an Antidepressant
}

\author{
Charles HK West ${ }^{*, '}$, James C Ritchie ${ }^{2}$ and Jay M Weiss' \\ 'Department of Psychiatry and Behavioral Sciences, Emory University School of Medicine, Atlanta, GA, USA; '2Department of Pathology, \\ Emory University School of Medicine, Atlanta, GA, USA
}

Neuropsychopharmacology (2010) 35, | 836-1837; doi: I 0.1 038/npp.20 I0.54

Sir

In this issue of the journal, we report that paroxetine (PAR), when given to young rats (adolescent age) in moderate doses, produced an increase in electrophysiological activity of locus coeruleus (LC) neurons early in the course of drug administration (West et al, 2010). This effect on LC activity is opposite to the usual decrease in LC activity produced by therapeutic regimens of antidepressant (AD) drug administration (ie, chronic administration of $\mathrm{AD}$ drugs). In contrast, the only effects seen in mature adult rats treated with PAR were the usual decreases in LC activity, with no increases seen at any dose or duration of treatment examined. Therefore, the LC response to PAR early in treatment of young animals suggests a countertherapeutic reaction that may relate to an increase in depressive symptomatology, and suicidal ideation in particular, said to occur in some adolescents early in treatment with PAR and other SSRIs.

Following submission of the article described above, we collected data from testing two additional AD drugs, one also associated with the risk of increased suicidality, the dual serotonin-NE reuptake inhibitor venlafaxine (VEN), and an $\mathrm{AD}$ minimally associated with such risk, the tricyclic desipramine (DMI), which has little capacity to block

* Correspondence: Dr CHK West, Department of Psychiatry and Behavioral Sciences, Emory University School of Medicine, Emory Briarcliff Campus, Building A, Room 504-N, 1256 Briarcliff Road, NE, Atlanta, GA 30306, USA, Tel: + I 404712 9770, Fax: + I 404712 9755, E-mail: cwest@emory.edu

Received 25 March 2010; accepted 26 March 2010 serotonin reuptake. A moderate dose of each drug (VEN: $10 \mathrm{mg} / \mathrm{kg}$ per day and DMI: $2.5 \mathrm{mg} / \mathrm{kg}$ per day) was administered to young rats for 4 days before recording, the duration of administration that produced the maximal increase in LC activity caused by PAR. As with PAR, these drugs were administered to young rats (45 days of age) through minipumps with an abdominal catheter attached for i.p. delivery. It should be noted that although the i.p. catheter was designed to facilitate transmission of vehicle from the pump and therefore reduce the possibility of pump output clogging, the i.p. catheter procedure also is more stressful than is standard subcutaneous minipump implantation, so that drug delivery by this method is accompanied by a higher degree of stress than is present with standard subcutaneous minipump delivery during the initial period of drug delivery. Thus, stress may well be a significant cofactor in producing the increase in LC activity seen early in treatment of young rats with PAR, although it is also quite clear that such stress does not cause LC activity of mature adult rats to be increased early in treatment with PAR or for LC activity to be increased in young rats administered vehicle by the same method.

The results from testing of VEN and DMI are shown in Figure 1. VEN produced a statistically significant increase in both spontaneous firing rate and sensory-evoked responses of LC neurons relative to vehicle-treated control rats. In contrast, DMI produced the opposite effect, a statistically significant decrease in both of these parameters of LC activity. Thus, an increase in LC activity with brief drug treatment of young animals has now been seen with two AD drugs that block 5-HT reuptake (PAR and VEN) and was not seen with a tricyclic $A D$ that is a very weak blocker of 5-HT reuptake (DMI). Consistent with the differential risk of these drugs for increasing depressive symptoms and suicidality early in treatment of young 

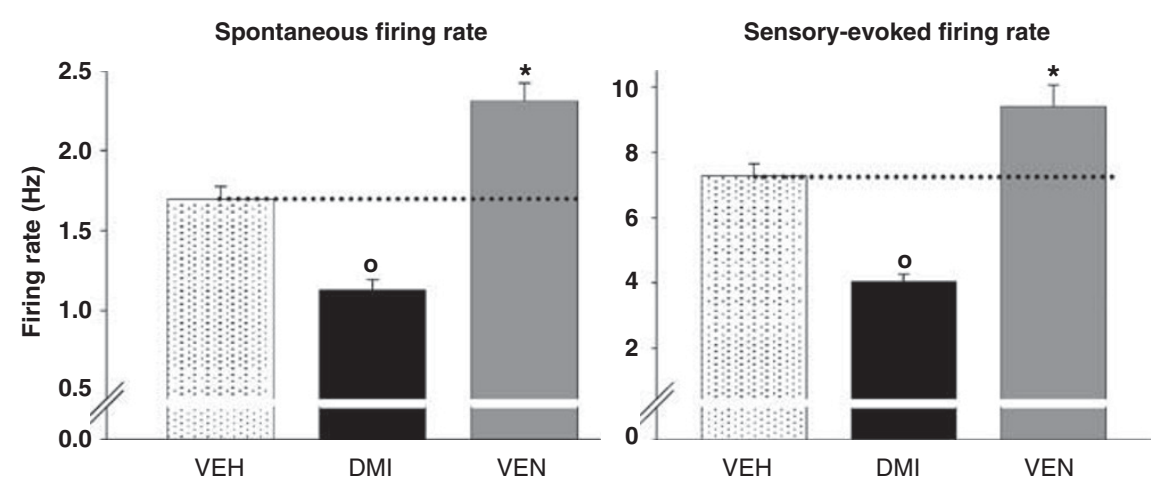

Figure I Spontaneous (left) and sensory-evoked (right) firing rate of LC neurons recorded in young rats (approximately 45 days of age) that had received vehicle (VEH), desipramine (DMl; $2.5 \mathrm{mg} / \mathrm{kg}$ per day) or venlafaxine (VEN; $10 \mathrm{mg} / \mathrm{kg}$ per day) for 4 days before recording. Mean(+SE) is shown. Data are from five animals in each condition ( 10 units recorded per rat). Symbols: o, significantly $(p<0.00 \mathrm{I})$ lower than VEH condition; *, significantly $(p<0.0 \mathrm{I})$ higher than VEH condition.

subjects, VEN had effects similar to those of PAR whereas DMI did not.

\section{DISCLOSURE}

The authors declare no conflict of interest.

\section{REFERENCES}

West CHK, Ritchie JC, Weiss JM (2010) Paroxetineinduced increase in activity of locus coeruleus neurons in adolescent rats: implication of a countertherapeutic effect of an antidepressant. Neuropsychopharmacology 35: 1653-1663. 